\title{
Understanding the Vibration Environment for LBT/AO
}

\author{
Pedro Escárate ${ }^{a^{*}}$, Julian C. Christou ${ }^{a}$, Gustavo Rahmer $^{a}$, Doug L. Miller ${ }^{a}$ and John M. Hill ${ }^{a}$ \\ ${ }^{a}$ Large Binocular Telescope Observatory, 933 North Cherry Ave, Tucson, AZ 85721 USA
}

\begin{abstract}
The majority of current Adaptive Optics (AO) systems do not properly compensate for vibrations within the optical path. For this reason the mitigation of vibration effects in astronomical AO systems is extremely important. A key component of AO operations is the implementation of techniques to improve the mitigation of vibrations in the AO system. We are in the process of characterizing the LBT AO vibration environment through frequency-based analysis of the closed-loop data, i.e. residual tip-tilt, and accelerometers measurements on key optical components. In this work we describe the vibration environment of the LBT and possible strategies to minimize the effect of the vibrations in the AO performance.
\end{abstract}

Keywords: Adaptive Optics, Power Spectral Density, Vibrations

\section{INTRODUCTION}

Adaptive Optics has become an essential technique to reach the highest image quality (Diffraction Limited) using deformable mirrors (DM) to mitigate the aberrations of astronomical images induced by the atmospheric turbulences. Currently the vast majority of 8-meter class telescopes are now equipped with adaptive optics systems and will be an integral component in the next generation of telescopes and astronomical instruments.

The vibration effects, acting in the propagation path of the science light, strongly affect the performance of the AO systems. Additionally, vibration often occurs at frequencies near or beyond the AO bandwidth, and therefore, may be poorly corrected.

The first evidence of the vibration problem were detected in the NAOS-CONICA at Paranal Observatory (Rousset et al, 20031] ; Clenet et al. 20042). During the commissioning of the instrument, a loss of performance of $15-20 \%$ was attributed to telescope vibrations. In the $8 \mathrm{~m}$ class telescopes high-frequency and large structural vibrations $(\geq 5 \mathrm{~Hz})$ are becoming more dominant in diffraction-limited observations.

The need to optimize the LBTO/AO system in terms of the atmospheric correction to reach the diffraction limit for the next generation of instrument to characterize exoplanets (e.g. SHARK ${ }^{\sqrt[8]{8}}$ ) is the actual motivation to mitigate the vibrations actually presesent in LBT.

In this work, a frequency domain characterization of the vibrations enviroment of LBT will be presented.

\section{VIBRATIONS MEASUREMENTS}

\subsection{LBT AO Model}

The structure for the AO system (Figure 1) used in his work to model the LBT/AO system (Escarate et al. $2017^{3}$ ) consists of a wavefront sensor (WFS), a deformable mirror (DM) and a controller (K). Assuming that the total disturbance incident phase $\varphi$ could be represented using the Zernike Polynomial decomposition, a different control rule can be designed for each independent wavefront mode following the structure presented in Figure 1. From this point of view, and without loss of generality, we have considered only tip and tilt modes of the Zernike Polynomial decomposition of the wavefront.

(*)Corresponding author: pescarate@lbto.org 


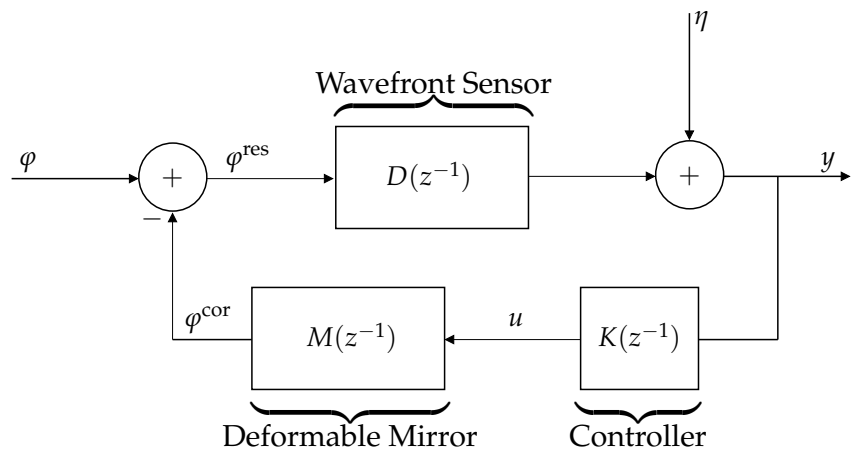

Figure 1: Block diagram for an AO system

In Figure 1, $\varphi, \varphi^{\mathrm{cor}}$, and $\varphi^{\mathrm{res}}$ represent the amplitudes for total disturbance phase, correction phase and residual phase, respectively. Total disturbance $\varphi$ is defined as the sum of the turbulence signal $\varphi^{\text {tur }}$ and vibration signals $\varphi_{t}^{\text {vib }_{i}}$. That is:

$$
\varphi_{t}=\varphi_{t}^{\mathrm{tur}}+\varphi_{t}^{\mathrm{vib}_{1}}+\varphi_{t}^{\mathrm{vib}_{2}}+\cdots+\varphi_{t}^{\mathrm{vib}_{n}} .
$$

The model describing the WFS measurement process and the DM correction process is assumed to be linear. For a sampling period $\Delta$, in this article we use a standard model for the WFS and DM, where their dynamics can be defined by the following discrete-time transfer functions:

$$
\begin{aligned}
D\left(z^{-1}\right) & =d_{0} z^{-1}, \\
M\left(z^{-1}\right) & =m_{0} z^{-1} .
\end{aligned}
$$

Thus, the output signal $y$ is given by:

$$
y_{t}=d_{0} \varphi_{t-1}^{\mathrm{res}}+\eta_{t}
$$

where $\eta_{t}$ is white Gaussian noise and

$$
\varphi_{t}^{\mathrm{cor}}=m_{0} u_{t-1} .
$$

In this article the residual phase $\varphi^{\text {res }}$ was used to estimate the vibrations not rejected by the LBT/AO control loop.

\subsection{OVMS}

The Optical path difference and Vibration Monitoring System (OVMS 4 ) is a system that currently estimates the tip-tilt based on a network of 45 acelerometers suitable for the frequency range $0.7-450 \mathrm{~Hz}$ (Figure 2 installed through the LBT structure working up to $4.5 \mathrm{kHz}$ sampling frequency. The OVMS system has five accelerometers on each of the telescope mirrors. Three of them make measurements in the Z-direction, which correspond to the direction of the optical axis for the primary and secondary mirror and the normal for the tertiary mirror face. The combined data is used to provide information on optical path length changes. The remaining two accelerometers per mirror measure along the perpendicular axes $\mathrm{x}$ and $\mathrm{y}$ and provides measurements of tip and tilt. Additionally, three roving accelerometers are available and can be placed at selected locations for test purposes. In order to make use of the accelerometers data, the Optical Path Difference (OPD) has to be calculated from the accelerometer measurements. For this, the LBT uses a software estimator called OVMS+ +5 designed to calculate piston, tip, and tilt values for all its main mirrors in real time. 


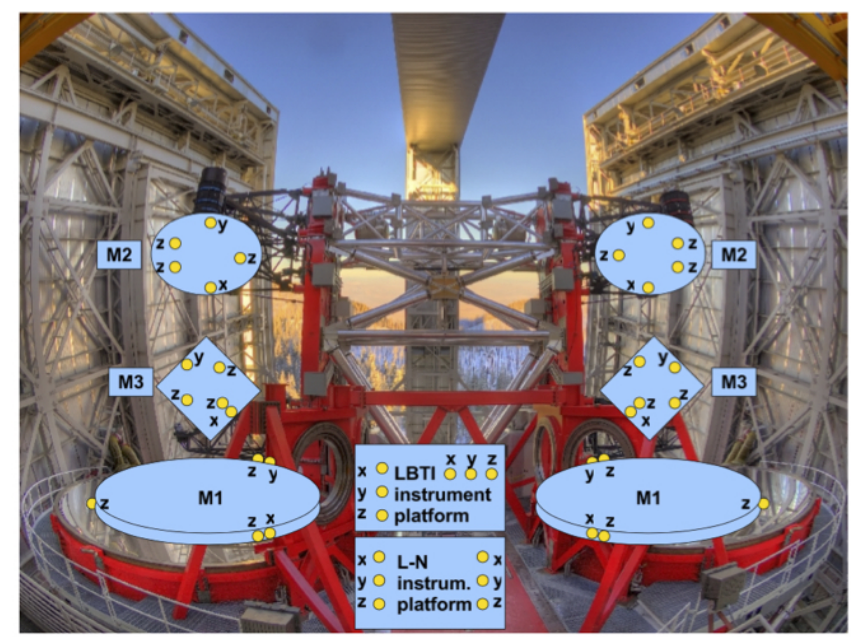

Figure 2: OVMS System accelerometer locations

\section{VIBRATION ANALYSIS}

\subsection{Wavefront Sensor Data}

Closed-loop data of the LBT/AO control loop from June 2016 to May 2017, was recorded and then used to calculate the Power Spectral Density (PSD) and the cumulative PSD of the residual phase $\left(\varphi^{\text {res }}\right)$. Figure 3 shows the PSD of the residual phase calculated for May 3rd, 2017.
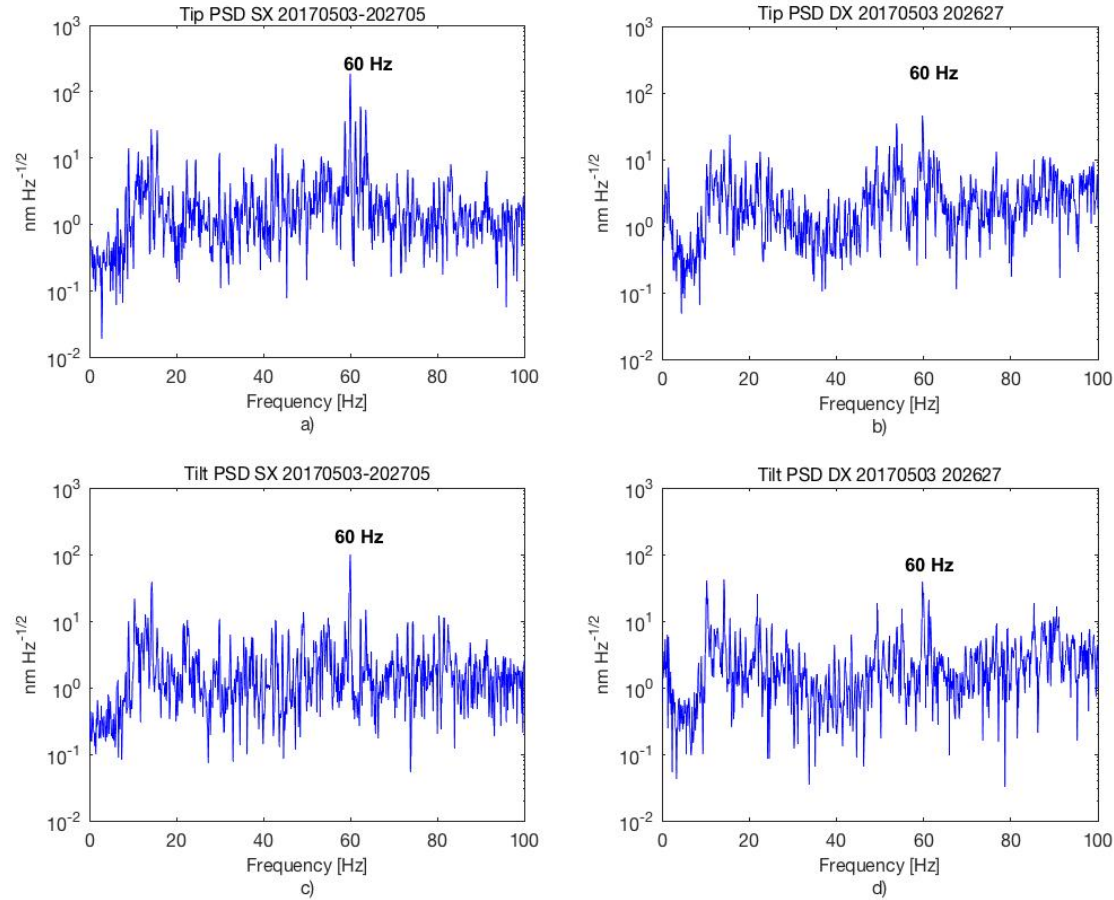

Figure 3: Vibration PSD measured in closed-loop. Left: LBT left side PSD. Right: LBT right side PSD. Upper:

Tip Mode PSD. Bottom: Tilt Mode PSDs 
The cumulative PSD was obtained in order to estimate the most important vibration peaks in term of their contribution to the total image jitter. The Figure 4 shows the cumulative PSD for May 3rd, 2017.
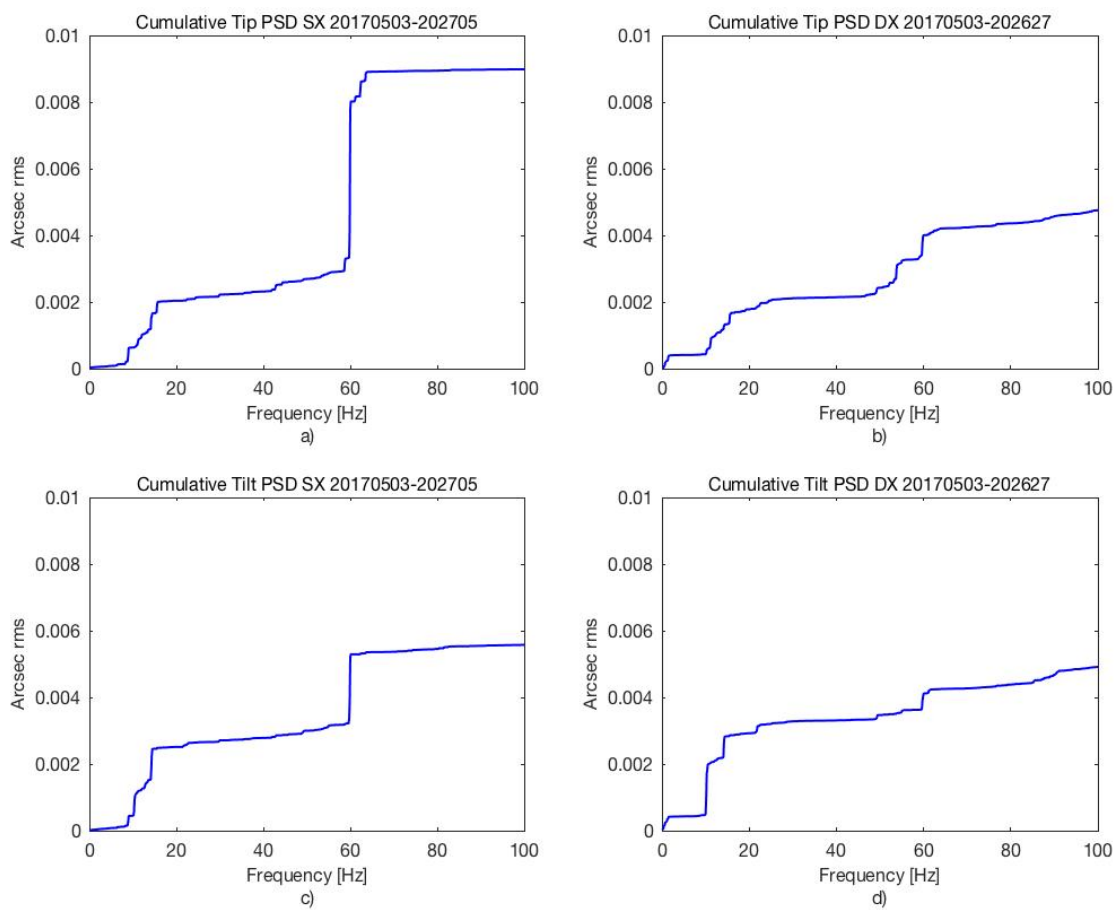

Figure 4: Vibration Cumulative PSD measured in closed-loop. Left: LBT left side Cumulative PSD. Right: LBT right side Cumulative PSD. Upper: Tip Mode Cumulative PSD. Bottom: Tilt Mode Cumulative PSD

The Figure 4 shows that there are 2 important vibration bands, one of them is around the $14[\mathrm{~Hz}]$ and the other one is around $60[\mathrm{~Hz}]$. These bands represent more than $95 \%$ of the total jitter in the final image.

\subsection{OVMS+ Data}

OVMS+ data from June 2016 to May 2017, was recorded and then used to calculate the Tip and Tilt PSD of the LBT optical path. Figures 5 and Figure 6 shows the PSD and cumulative PSD estimated using the OVMS+ system for May 3rd, 2017. 

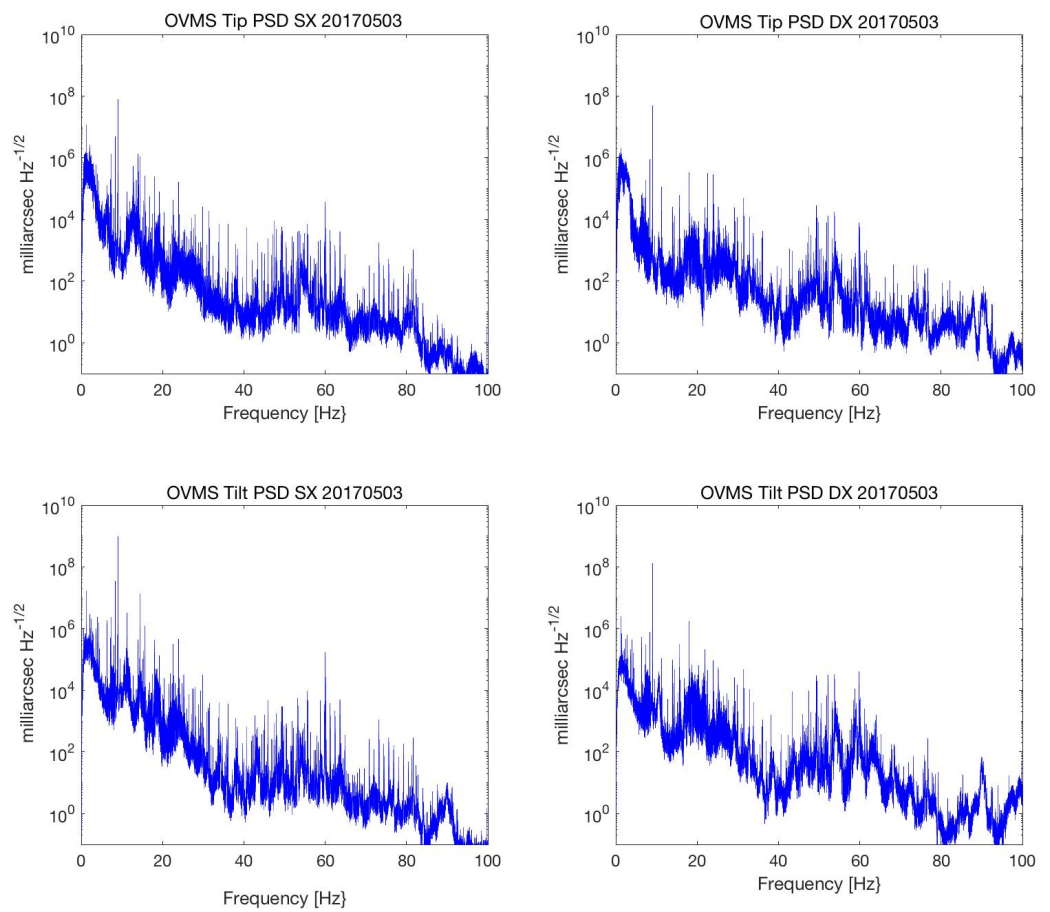

Figure 5: Vibration measured using OVMS+ system. Left: LBT left side PSDs. Right: LBT right side PSDs. Upper: Tip Mode PSDs. Bottom: Tilt Mode PSDs
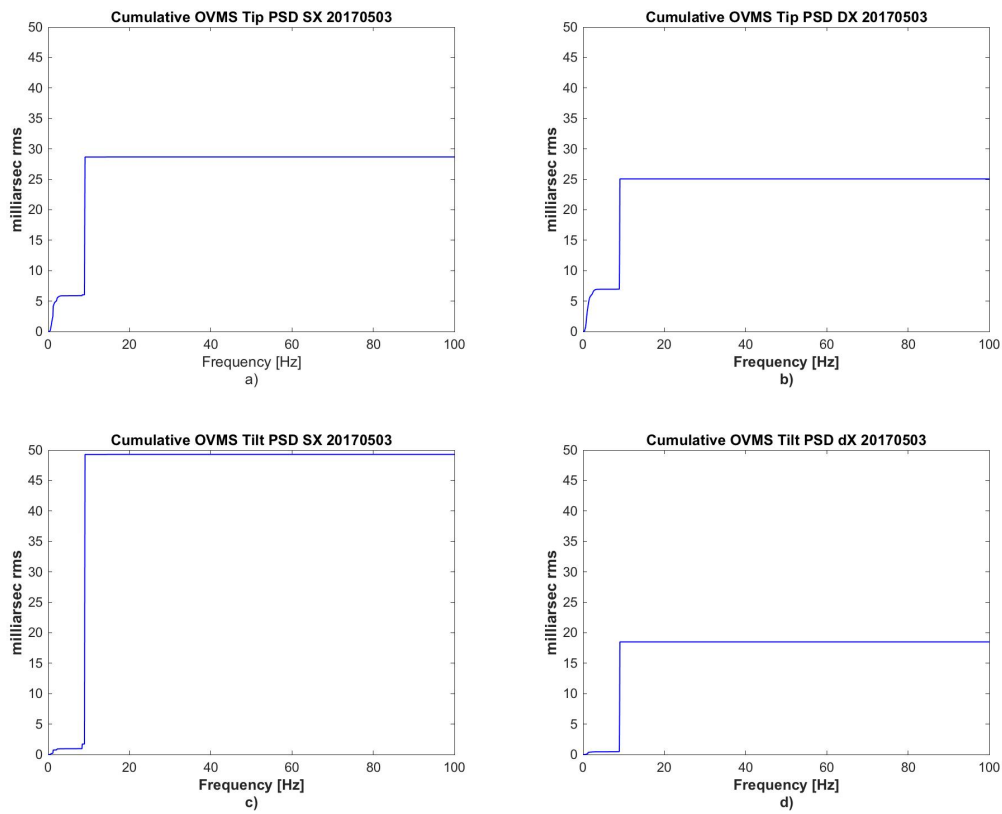

Figure 6: Cumulative PSD OVMS+ system. Left: LBT left side PSDs. Right: LBT right side PSDs. Upper: Tip Mode PSDs. Bottom: Tilt Mode PSDs 
Figures 5 and 6 shows that there are several vibration peaks, althought it is still possible to see vibration peaks in the same bands than the residual phase $\left(\varphi^{\text {res }}\right)$ around $14[\mathrm{~Hz}]$ and $60[\mathrm{~Hz}]$.

\section{CONCLUSIONS AND FUTURE WORK}

The analisys presented in Section 3 illustrate the vibration enviroment of the LBT and the effect of the vibrations on the LBT/AO system.

The Figures in Section 3 shows that there are 2 important vibration bands detected in both systems that represents more that the $90 \%$ of the jitter produced by the vibrations. The first of them is around 14 [Hz] (Figure 7) and the other around $60[\mathrm{~Hz}]$ (Figure 8). Also is possible to see that the $60[\mathrm{~Hz}]$ band produces more jitter than the $14[\mathrm{~Hz}]$ band and that the $99 \%$ of the jiiter detected by OVMS+ Tip and tilt is on 0-20 [Hz] band.
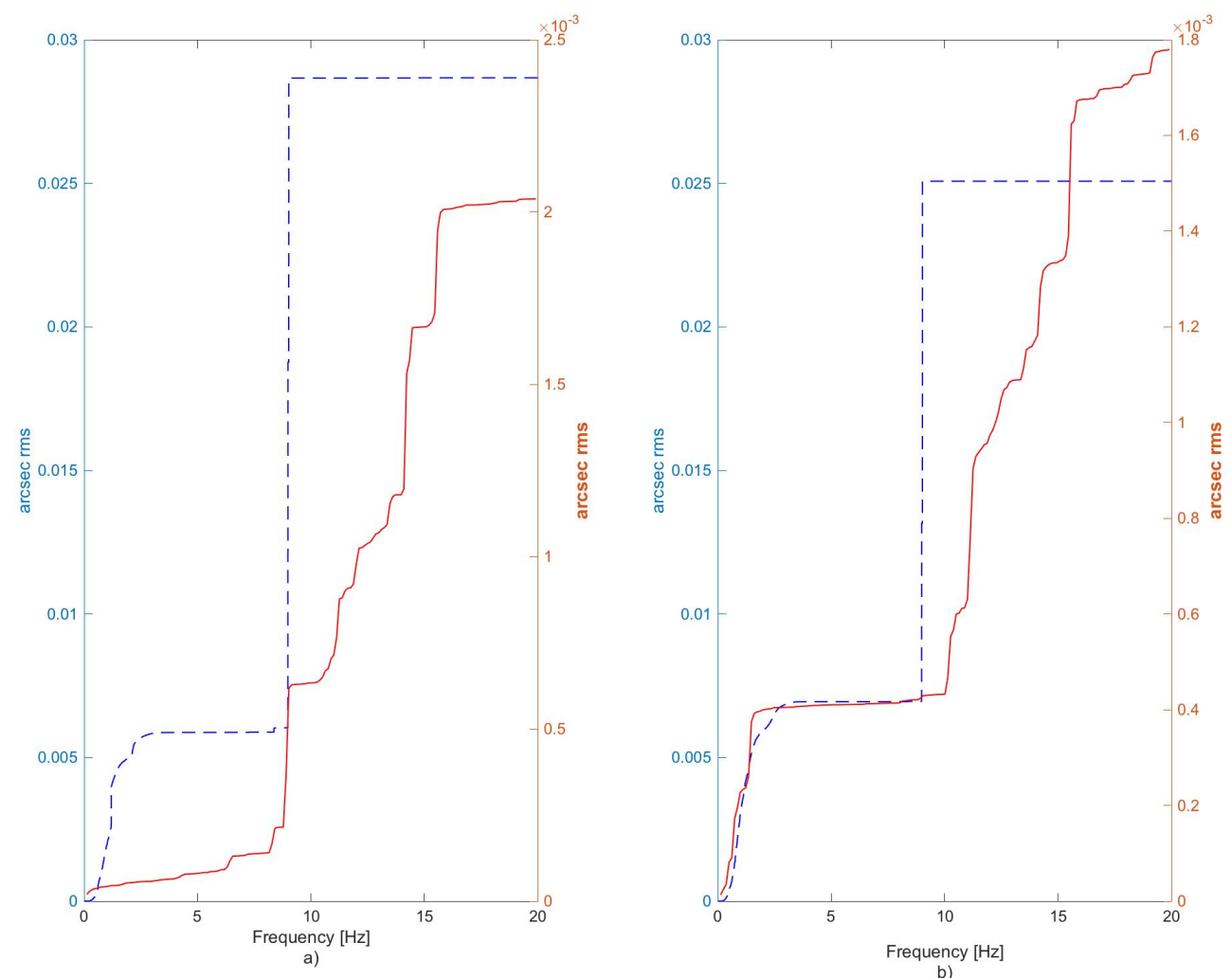

Figure 7: $14[\mathrm{~Hz}]$ band zoom. The blue line respresents the OVMS+ Cumulative PSD and the red line represent the Residual Phase Cumulative PSD. a) Left side PSD. b) Right side PSD. 

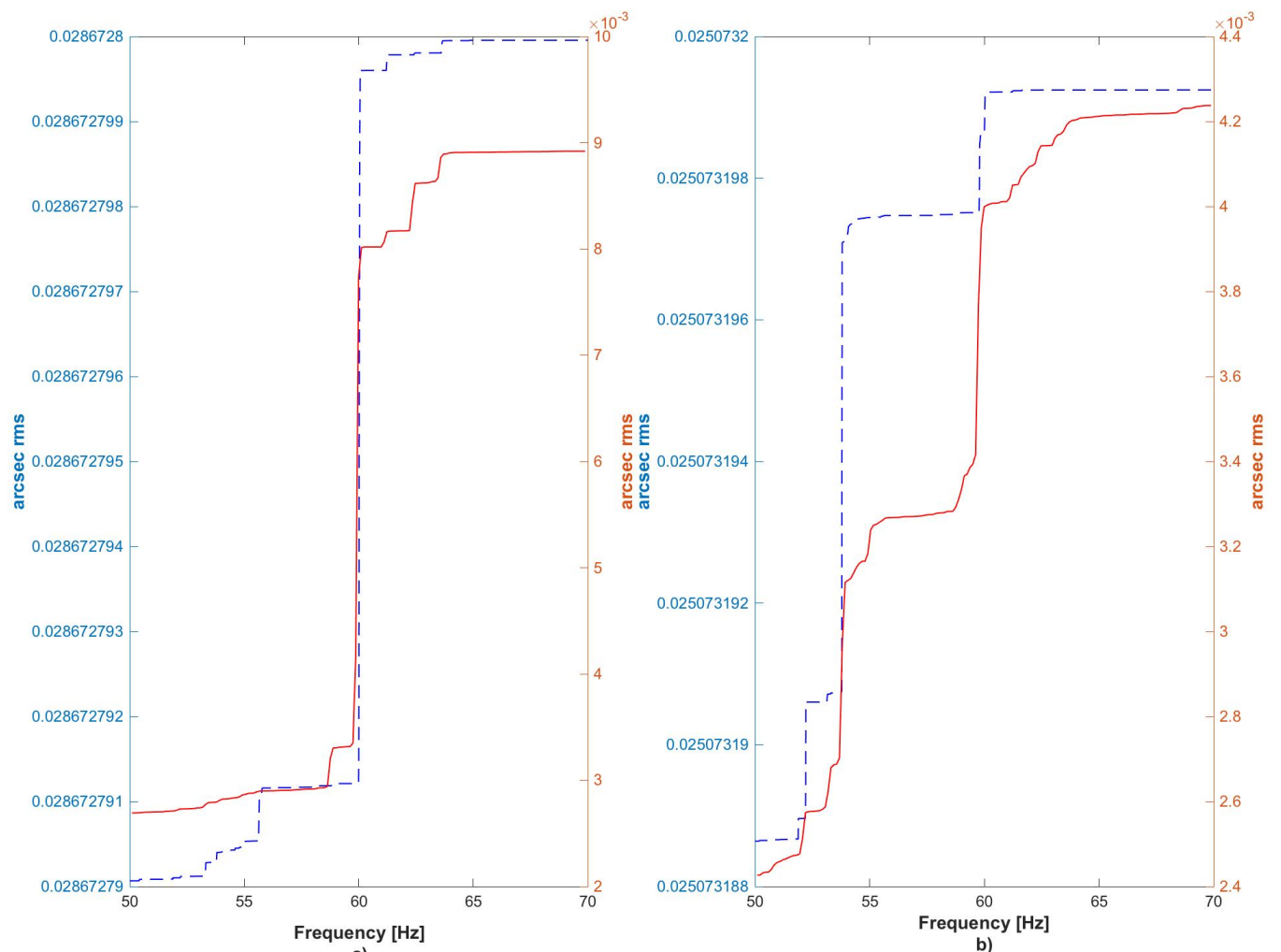

Figure 8: $60[\mathrm{~Hz}]$ band zoom. The blue line respresents the OVMS+ Cumulative PSD and the red line represent the Residual Phase Cumulative PSD. a) Left side PSD. b) Right side PSD.

Figures 7 and 8 show that there is a high correlation between the vibration peaks detected by the OVMS+ and the residual phase $\left(\varphi^{\mathrm{res}}\right)$. Althought the both system are in different scales, and the direct effect in the AO system of the vibration detected by the OVMS+ system is not clear.

Finally using this information it is possible to investigate the sources of those vibration peaks or to implement techniques to mitigate the effect of the vibration in the LBT/AO system. For future work, the LBT/AO system can be tested using advanced control strategies in order to mitigate the effect of the vibrations. 


\section{REFERENCES}

1. Gerard Rousset, Francois Lacombe, Pascal Puget, Norbert N. Hubin, Eric Gendron, Thierry Fusco, Robin Arsenault, Julien Charton, Philippe Feautrier, Pierre Gigan, Pierre Y. Kern, Anne-Marie Lagrange, PierreYves Madec, David Mouillet, Didier Rabaud, Patrick Rabou, Eric Stadler, Gerard Zins, "NAOS, the first ao system of the vlt: on-sky performance," Proc. SPIE 4839, pp. 4839 - 4839 - 10, 2003.

2. Yann Clenet, Markus E. Kasper, Nancy Ageorges, Christopher Lidman, Thierry Fusco, Olivier Marco, Markus Hartung, David Mouillet, Bertrand Koehler, Gerard Rousset, Norbert N. Hubin, "NACO performance: status after 2 years of operation," Proc. SPIE 5490,pp. 5490 - 5490 - 11, 2004

3. Pedro Escárate, Rodrigo Carvajal, Laird Close, Jared Males, Katie Morzinski and J. C. Agüero, "Minimum variance control for mitigation of vibrations in adaptive optics systems," Applied Optics 56(19), pp. 53885397, 2017.

4. M. Kürster, T. Bertram, J.L. Borelli, M. Brix, W. Gässler, T.M. Herbst, V. Naranjo, J.U. Pott, J. Trowitzsch, T.E. Connors, P.M. Hinz, T.J. McMahon, D.S. Ashby, J.G. Brynnel, N.J. Cushing, T. Edgin, J.D. Esguerra, R.F. Green, J. Kraus, J. Little, U. Beckmann, G.P. Weigelt, "OVMS: the optical path difference and vibration monitoring system for the LBT and its interferometers," Proc. SPIE 7734, pp. $7734-7734-8,2010$.

5. Michael Böhm, Jörg-Uwe Pott, José Borelli, Phil Hinz, Denis Defrère, Elwood Downey, John Hill, Kellee Summers, Al Conrad, Martin Kürster, Tom Herbst, Oliver Sawodny, "Ovms-plus at the lbt: disturbance compensation simplified," Proc. SPIE 9906, pp. 9906 - 9906 -8, 2016.

6. Javier Garcés, Sebastián Zuñiga, Laird M. Close, Jared R. Males, Katie M. Morzinski, Pedro Escárate and Mario Castro, "Vibrations in MagAO: resonance sources identification through frequency-based analysis," Imaging and Applied Optics 2015, 2015.

7. Sebastián Zuñiga, Javier Garcés, Laird M. Close, Jared R. Males, Katie M. Morzinski, Pedro Escárate and Mario Castro, "Vibrations in MagAO: analysis of on-sky data and future challenges in vibrations mitigation," Imaging and Applied Optics 2015, 2015.

8. Jacopo Farinato, Fernando Pedichini, Enrico Pinna, Francesca Baciotti, Carlo Baffa, Andrea Baruffolo, Maria Bergomi, Pietro Bruno, Enrico Cappellaro, Luca Carbonaro, Alexis Carlotti, Mauro Centrone, Laird Close, Johanan Codona, Silvano Desidera, Marco Dima, Simone Esposito, Daniela Fantinel, Giancarlo Farisato, Adriano Fontana, Wolfgang Gaessler, Emanuele Giallongo, Raffaele Gratton, Davide Greggio, Juan Carlos Guerra, Olivier Guyon, Philip Hinz, Francesco Leone, Franco Lisi, Demetrio Magrin, Luca Marafatto, Matteo Munari, Isabella Pagano, Alfio Puglisi, Roberto Ragazzoni, Bernardo Salasnich, Eleonora Sani, Salvo Scuderi, Marco Stangalini, Vincenzo Testa, Christophe Verinaud, Valentina Viotto, "SHARK (System for coronagraphy with High order Adaptive optics from R to K band): a proposal for the LBT 2nd generation instrumentation," Proc. SPIE 9147, pp. 9147 - 9147 - 10, 2014. 